\title{
Anti-TNF-Therapie bald auch bei Arthrose?
}

Zur Arthrose-Therapie liegen erste Daten mit TNFa-Hemmern vor. Chondroitinsulfat lindert bei Gonarthrose die Schmerzen ähnlich gut wie ein Coxib. In der Entwicklung befindet sich rein peripher wirkendes Fentanyl.

In einer Pilotstudie wurden fünf Patienten mit erosiver Handarthrose radioaktiv-markiertes Technecium-99 m-Certolizumab injiziert, das an TNFa bindet. Die Szintigrafie zeigte eine Aufnahme der Substanz in 24 kleinen Gelenken. Gelenke mit Weichteilschwellung nahmen das Tc-99 m-Certolizumab stärker auf als nicht geschwollene, so Prof. Dr. Christoph Baerwald, Uniklinikum Leipzig.

In einer Interventionsstudie führte die Gabe von Etanercept allerdings nicht zu einer Reduktion der Schmerzen bei Patienten mit Hand-Polyarthrose. Im MRT zeigte sich hinsichtlich der Knochenmarksläsionen ein deutlicher Unterschied: Diese nahmen in den Gelenken mit Synovitis mit dem TNFaBlocker deutlich ab, unter Placebo hingegen deutlich zu. Und eine Analyse aus der BeSt-Studie ergab, dass jeder Monat einer anti-TNFa-Therapie das relative Risiko einer Arthrose-Progression in den DIP-Gelenken mindert.,Die Rolle von TNFa bei Arthrose ist auch weiterhin unklar", schloss Baerwald.

Therapieziel bei Polyarthrose der Hand ist die Symptomlinderung, nach den EULAR-Empfehlungen u.a.:

- Orthesen bei Daumengrundgelenksarthrose können in Betracht gezogen werden - die Langzeitnutzung wird empfohlen

- Topische NSAR sind erste Wahl der Pharmakotherapie

- Intraartikuläre GlucokortikoidInjektionen können bei schmerzvollen Interphalangeal-Gelenken in Betracht kommen

- Chondroitinsulfat kann zu Schmerzlinderung und Funktionsverbesserung eingesetzt werden.

Zum Chondroitinsulfat (CS) präsentierte Baerwald Daten einer dreiar- migen Studie. Darin erhielten rund 600 Gonarthrosepatienten entweder $800 \mathrm{mg} / \mathrm{d}$ CS, $200 \mathrm{mg} / \mathrm{d}$ Celecoxib oder Placebo. In Bezug auf Schmerzen und Funktionsindex gab es keine Unterschiede zwischen den beiden Verumgruppen. Unter Verum nahmen die Schmerzen auf der Skala mit 100 Punkten um 43 (CS), 40 (Coxib) und 33 Punkte ab (Placebo). Den Patienten solle pharmakologisch hergestelltes Chondroitinsulfat empfohlen werden, riet Baerwald.

Möglicherweise wird ein neues Opioid die Schmerztherapie erweitern. Hier liefen Versuche mit einem speziellen Fentanyl (NFEPP). Darin ist Wasserstoff durch Fluor ersetzt, wodurch das Opioid nur im sauren
Milieu an den $\mu$-Opioidrezeptor bindet - also dort wo die Entzündung in den Gelenken stattfindet. Das Spezial-Fentanyl habe daher nur periphere, keine zentralen Effekte, so Baerwald. Allerdings befinde sich die Forschung hier noch in der Phase der Tierexperimente. Zum breit debattierten Thema Cannabis merkte der Rheumatologe an, dass die Daten in der Schmerztherapie eher dünn seien. „Ausnahme ist hier der neuropathische Schmerz."

rheuma plus $2019 \cdot 18: 105$ https://doi.org/10.1007/s12688-0190260-0

(c) Springer-Verlag GmbH Austria, ein Teil von Springer Nature 2019 